\title{
Macro- and micro-cracking in one-dimensional elasticity
}

\author{
Gianpietro Del Piero ${ }^{a}$, Lev Truskinovsky ${ }^{b, *}$ \\ a Dipartimento di Ingegneria, Universita' di Ferrara, Via Saragat 1, 44100 Ferrara, Italy \\ ${ }^{\mathrm{b}}$ Department of Aerospace Engineering and Mechanics, University of Minnesota, 110, Union Street, 107, Akerman Hall, Minneapolis, \\ MN 55455, USA
}

Received 12 June 1999; in revised form 15 February 2000

\begin{abstract}
In classical fracture mechanics, the equilibrium configurations of an elastic body are obtained by minimizing an energy functional containing two contributions, bulk and surface. Usually, the bulk energy is convex and the surface energy is concave. While this type of minimization successfully describes macroscopic cracks, it fails to model microdefects forming a so-called process zone. To describe this phenomenon, we consider, in this paper, a model with a nonconcave, "bi-modal" surface energy, which allows the formation of both macro- and micro-cracks. Specifically, we consider the simplest one-dimensional problem for a bar in a hard device and show that if the surface energy is not subadditive, the solution exhibits a new mode of failure with a finite number of micro-cracks coexisting with one fully developed macro-crack. We present an explicit example of a "quantized" micro-cracking with a subsequent development into a single macro-crack. () 2001 Elsevier Science Ltd. All rights reserved.
\end{abstract}

Keywords: Macro-cracking; Micro-cracking; One-dimensional elasticity

\section{Introduction}

Separation of elastic energy into bulk and surface (cohesive) parts is a classical assumption in phenomenological fracture mechanics. The bulk energy is a function of strain and the cohesive energy is a function of the components of relative displacements on the surface of discontinuity. This assumption was introduced by Griffith (1920) and modified later by Barenblatt (1962) to account for the cohesive forces which oppose fracture opening. Traditionally, the bulk energy is taken to be quadratic, while the surface energy is assumed to be concave. Fracture models with convex bulk energy and concave cohesive energy successfully reproduce localized fracture. Thus, for a homogeneous bar subject to a prescribed elongation, the minimum of the total energy corresponds to a configuration with a single crack.

The mechanism of the energy separation into bulk and surface parts can be understood from the perspective of discrete models with Lennard-Jones type interaction. The corresponding continuum limit was

\footnotetext{
${ }^{*}$ Corresponding author. Tel.: +1-612-624-0529; fax: +1-612-626-1558.

E-mail address: trusk@aem.umn.edu (L. Truskinovsky).
} 
studied by Truskinovsky (1996) and Braides et al. (1999) for a one-dimensional lattice with nearest neighbors interaction. It was shown that for a sufficiently large number of interacting particles, the discrete model can be approximated by a continuum model with a convex bulk energy and a concave cohesive energy. As one would expect, these two energies represent different segments of the original non-convex interatomic potential.

Although the macro-cracking in brittle materials is usually responsible for the major part of the energy release, one also observes a less localized damage in the vicinity of the tip of the crack and at the stage preceding the nucleation of the macro-crack. The presence of the distributed damage coexisting with a main crack is usually attributed to micro-cracking or micro-debonding of pre-existing defects. It is known that the micro-crack opening in the "process zone" is taking place at a considerably smaller scale than in the case of a macro-crack. Sometimes, the micro-cracking is modeled in the classical framework, although not in a self-consistent way which would illuminate the energetic necessity and mechanical stability of distributed micro-cracking (Kachanov, 1993).

In this paper, we suggest a new model of brittle micro-damage, which is based on a "bi-modality" of the cohesive energy. Specifically, we assume that the process of creation of the crack can be decomposed into two steps: nucleation of micro-defects and the subsequent opening of a macro-crack. The surface energy as a function of the relevant component of the crack opening is then assumed to possess two, rather than one, plateaus: a small one, near the origin, and a major one which extends to infinity. The corresponding forceopening relation is characterized by two peaks: the one near the origin and the other one away from the origin. The important mathematical consequence of this assumption is that the surface energy is no longer concave on the whole interval but instead has a convex domain located between two concave domains. In what follows, we focus on the analysis of the corresponding model and on the demonstration of the main effects associated with the non-concavity of the surface energy. A couple of remarks of physical origin, however, are in order.

The peculiar behavior of the surface energy may be related to two mechanisms. First notice that, due to the presence of long range interactions, the crack opening is accompanied by a local lattice distortion near the free surface: the corresponding boundary layers are neglected in the continuum model which does not contain any internal length scale. This observation suggests that there may be two characteristic levels of crack opening and two surface energies: one, associated with the formation of the corresponding boundary layers ("scars"), and another one associated with the ultimate separation of the new, fully developed interfaces. It is natural to think that the formation of the "scars" precedes the opening of a macroscopic crack.

The second reason for the "bi-modality" of the surface energy may be traced to the fact that, microscopically, the material may be built of two types of elements: "strong" and "weak". A certain finite energy is required to break the "weak" micro-elements while a much larger energy is necessary to break the "strong" elements responsible for a visible displacement discontinuity. Breaking of the "weak" elements may be associated with debonding of the micro inclusions, creation of the staking faults or twins and, possibly, with the occurrence of phase transitions. One can also think about initial "point" cavitation, which precedes the opening of the surfaces of discontinuity (see Baljon and Robbins (1996)).

The evidence that the non-concavity of the surface energy may give rise to multiple cracking was, although indirectly, provided by Braides and Coscia (1993) and Del Piero (1999). Working with linear or convex surface energies, these authors showed that in the one-dimensional problem, the energy minimum can be attained at a configuration with an infinite number of infinitesimal cracks (diffuse zones of nondifferentiability). This situation can be described in the context of the theory of structured deformations of Del Piero and Owen (1993), and was associated in Del Piero (1999) with plastic-like behavior. In the case of a linear surface energy, Braides and Coscia (1993) also considered a regularized problem with a finite surface energy threshold. 
In the present paper, we discuss in some detail a one-dimensional model with a cohesive energy, which is convex on a finite segment separated from the origin and concave outside. As the total elongation increases, this model predicts the formation of a finite number of micro-cracks, one after another, until a macro-crack forms. This regime of distributed micro-cracking can be viewed as a "quantized" propagation of damage. The resulting total energy turns out to be a non-smooth function of the average strain. It can be represented by a finite number of convex curves, each corresponding to a configuration with a fixed number of cracks. The overall stress-strain relation is then discontinuous and has a characteristic sawtooth structure (for similar effects in martensites see Truskinovsky and Zanzotto (1996)). When the concave region near the origin shrinks to zero, the model recovers "structured deformations", and when the convex region disappears, a localized fracture appears as another limiting case.

The structure of the paper is as follows. In Section 2, we formulate the one-dimensional model and introduce our assumptions concerning the bulk and surface energies. In Section 3, we derive necessary and sufficient conditions for metastability. The structure of global minimizers is investigated in Section 4, where we also formulate conditions on the surface energy which guarantee the presence of a finite number of micro-cracks in addition to a macro-crack. The simplest example of a surface energy leading to quantized micro-cracking is worked out in closed form in Section 5.

Some of the preliminary results of this work were announced in Del Piero and Truskinovsky (1998).

\section{The model}

Consider a homogeneous elastic bar of reference length $l$, with strain energy of the form

$$
E\{y\}=\int_{0}^{l} w\left(y^{\prime}(x)\right) \mathrm{d} x+\sum_{k=1}^{N} \theta\left([y]\left(x_{k}\right)\right),
$$

where $w$ and $\theta$ are the bulk and surface energies, respectively, $y:[0, l] \rightarrow \mathbf{R}$ is the axial deformation of the bar, $y^{\prime}$ is the deformation gradient, $[y]\left(x_{k}\right)$ is the jump discontinuity of $y$ at the jump point $x_{k}$, and $N$ is the number of jump points. We assume that the function $y$ satisfies the non-interpenetration assumptions

$$
y^{\prime}>0, \quad[y] \geqslant 0 .
$$

We also suppose that the bar is loaded in a hard device, which means that the total extension is prescribed

$$
\int_{0}^{l} y^{\prime}(x) \mathrm{d} x+\sum_{k=1}^{N}[y]\left(x_{k}\right)=d .
$$

Here, $d$ is the length of the bar after deformation. The mathematical problem consists in minimizing the functional (2.1) under the constraints (2.2) and (2.3) and includes the determination of the jump points of the solution $y$.

Motivated by the physical setting of the problem, we shall be interested in studying both global and local minima (stable and metastable configurations). Local minima will be understood in the sense of the supnorm rather than in the sense of some integral norm. This implies, e.g., that for a perturbation to be small, the newly formed displacement discontinuity must be infinitesimal. This specific choice of the class of competitors does not affect the global minimum, but is crucial in deciding which state has to be considered as metastable.

The function $w:(0, \infty) \rightarrow(0, \infty)$, representing the bulk energy, is assumed to be strictly convex and to satisfy

$$
w(1)=w^{\prime}(1)=0 .
$$



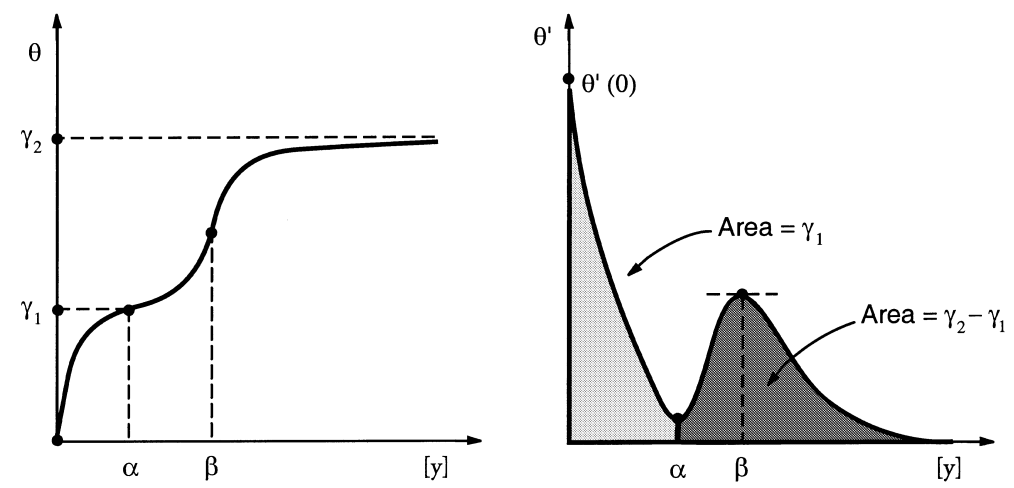

Fig. 1. Bi-modal (concave-convex-concave) surface energy $\theta$ and the corresponding force-opening relation $\theta^{\prime}$. Parameters $\gamma_{1}$ and $\gamma_{2}$ represent the energies of micro- and macro-cracking.

The assumption of convexity eliminates from the picture phenomena like strain localization and phase transitions, and allows us to focus on fracture. Without any substantial loss of generality, we may assume that $w$ is quadratic, like in classical linear fracture mechanics.

The cohesive or interfacial energy $\theta:[0, \infty) \rightarrow[0, \infty)$ is assumed to satisfy

$$
\theta(0)=0, \quad \theta^{\prime}(0)>0 .
$$

The first of these conditions suggests that the energy barrier against the formation of an infinitesimal discontinuity is equal to zero, while the second condition states that the corresponding stress barrier is positive.

It is usually assumed that the function $\theta$ is concave. Thus, in the model of Griffith (1920), the surface energy is constant everywhere except at the origin, where it is equal to zero. The "cohesive" model of Barenblatt (1962) assumes that a surface energy is continuous and concave. In what follows, we focus on surface energies which are not concave, and we formulate a variety of results applicable to models with surface energies having arbitrary convexity-concavity properties.

The typical "bi-modal" surface energy, described in the introduction, is sketched in Fig. 1, where we also show the corresponding force-opening relation. The two parameters $\gamma_{1}$ and $\gamma_{2}$ are the energies required to create a micro- and a macro-crack, respectively. The presence of the domain of convexity of $\theta$ between the inflection points $\alpha$ and $\beta$ determines the non-monotonicity of the force-opening relation; when points $\alpha$ and $\beta$ coincide and the force-elongation relation is monotonic, we obtain the standard "cohesive zone" model. Notice that the areas under the maxima of the force-opening curve determine the fracture energies $\gamma_{1}$ and $\gamma_{2}$. The important aspect of the "bi-modal" surface energy is that it is not concave and may not even be subadditive (seen below), which turns out to be necessary for the existence of multiple cracking.

\section{Equilibrium and stability}

We begin by investigating some necessary conditions for equilibrium, and show that our infinite dimensional variational problem can be reduced to a finite dimensional problem. Let us consider an arbitrary deformation $y$ and compare it with a piecewise linear deformation $\bar{y}$, having the same discontinuities as $y$ and satisfying the condition $\bar{y}^{\prime}(x)=$ const. The magnitude of the constant is chosen in such a way that the two deformations have the same average deformation gradient 


$$
\bar{y}^{\prime}=l^{-1} \int_{0}^{l} y^{\prime}(x) \mathrm{d} x
$$

In view of the convexity of $w$, we may apply Jensen's inequality to obtain

$$
E(y)-E(\bar{y})=\int_{0}^{l}\left(w\left(y^{\prime}(x)\right)-w\left(\bar{y}^{\prime}\right)\right) \mathrm{d} x \geqslant l w\left(l^{-1} \int_{0}^{l} y^{\prime}(x) \mathrm{d} x\right)-l w\left(\bar{y}^{\prime}\right)=0 .
$$

Accordingly, we can relax the energy by replacing the strain field $y^{\prime}$ with a constant field $\bar{y}^{\prime}$. Introduce the notation

$$
y^{\prime}=p, \quad[y]\left(x_{k}\right)=q_{k},
$$

where $k=1, \ldots, N$, and $N$ is the (unknown) number of jumps of $y$. Then the original infinite dimensional minimum problem reduces to the finite dimensional problem

$$
\begin{aligned}
& \bar{E}\left(p, q_{k}\right)=l w(p)+\sum_{k=1}^{N} \theta\left(q_{k}\right) \rightarrow \min , \\
& l p+\sum_{k=1}^{N} q_{k}=d, \quad p>0, \quad q_{k}>0 .
\end{aligned}
$$

This problem is identical with the equilibrium problem for a discrete chain containing $N+1$ non-linear springs in series, placed in a hard device with a prescribed total displacement. In this new framework, cracks are represented by springs with energies $\theta\left(q_{k}\right)$, where $q_{k}$ is the deformed length of the $k$-th spring. The bulk phase is represented by the $N+1$-th spring with deformed length $q=l p$ and with energy

$$
\Theta(q)=l w\left(q l^{-1}\right) .
$$

For the discrete chain, the equilibrium problems (3.3) and (3.4) can be written as

$$
\begin{aligned}
& \hat{E}\left(q, q_{k}\right)=\Theta(q)+\sum_{k=1}^{N} \theta\left(q_{k}\right) \rightarrow \min , \\
& q+\sum_{k=1}^{N} q_{k}=d, \quad q>0, \quad q_{k}>0 .
\end{aligned}
$$

Although the problem now looks quite elementary, a complication arises from the fact that the energies of all springs except one are non-convex and that the number $N$ of the springs is unknown.

It is natural to solve the problems (3.6) and (3.7) in two stages. First, one can assume that the number of discontinuities $N$ is prescribed, so that the problem reduces to finding the corresponding $q$ and $q_{k}$. The second stage will then consist of integer minimization in $N$.

The problem of a discrete chain with $N$ bi-stable springs was studied in Puglisi and Truskinovsky (2000), and we can use some of their results. Obviously, a necessary condition for equilibrium is the equality of "forces" acting inside different springs,

$$
\Theta^{\prime}(q)=\theta^{\prime}\left(q_{k}\right), \quad k=1, \ldots, N .
$$

To obtain a stability condition in this finite-dimensional context, we use Eq. (3.7) to rewrite Eq. (3.6) as an unconstrained problem for $N$ variables $q_{k}$ 


$$
\tilde{E}\left(q_{k}\right)=\Theta\left(d-\sum_{k=1}^{N} q_{k}\right)+\sum_{k=1}^{N} \theta\left(q_{k}\right) \rightarrow \min .
$$

The Hessian matrix for this problem takes the form

$$
\mathbf{H}=\left\|\begin{array}{cccc}
A+B_{1} & A & \ldots & A \\
A & A+B_{2} & \ldots & \ldots \\
\ldots & \ldots & \ldots & \ldots \\
A & \ldots & \ldots & A+B_{N}
\end{array}\right\|,
$$

where $A=\Theta^{\prime \prime}(q)>0$ and $B_{k}=\theta^{\prime \prime}\left(q_{k}\right)$. The main minors of this matrix can be calculated explicitly (see Puglisi and Truskinovsky (2000)), and we obtain the condition of stability in the form

$$
\left(\prod_{k=1}^{n} B_{k}\right)\left(\frac{1}{A}+\sum_{k=1}^{n} \frac{1}{B_{k}}\right) \geqslant 0 .
$$

Here $n=1, \ldots, N$, so we have $N$ conditions. According to Eq. (3.10), if the surface energy is convex and if $B_{k}>0$ for all $k$, then any equilibrium configuration is a minimizer. Since this is not the case, we have to continue our analysis. For $n=1$ we obtain

$$
\Theta^{\prime \prime}(q)+\theta^{\prime \prime}\left(q_{k}\right) \geqslant 0 \text {. }
$$

for any $k=1, \ldots, N$. At $n=2$, we get

$$
\Theta^{\prime \prime}(q)\left[\theta^{\prime \prime}\left(q_{k}\right)+\theta^{\prime \prime}\left(q_{l}\right)\right]+\theta^{\prime \prime}\left(q_{k}\right) \theta^{\prime \prime}\left(q_{l}\right) \geqslant 0,
$$

for every $k, l$ in $\{1, \ldots, N\}$ with $k \neq l$. The two inequalities (3.11) and (3.12) can be combined to get

$$
\theta^{\prime \prime}\left(q_{k}\right)+\theta^{\prime \prime}\left(q_{l}\right) \geqslant 0, \quad k \neq l,
$$

which implies that a minimizer cannot contain more than one crack with the opening located in the domain of concavity of the surface energy (where $\theta^{\prime \prime}\left(q_{k}\right)<0$ ). In case there exists exactly one such crack, the only non-trivial stability condition can be written as

$$
\left(\frac{1}{A}+\sum_{k=1}^{N} \frac{1}{B_{k}}\right) \leqslant 0
$$

This inequality has a simple geometrical interpretation. It is known that, if $N$ linear springs with elastic moduli $k_{i}$ are connected in series, the effective elastic modulus of the whole chain equals $\left(\sum k_{i}^{-1}\right)^{-1}$. In our case, considering $A$ and $B_{k}$ as tangent moduli at the given equilibrium configuration, we obtain

$$
\frac{\mathrm{d} \sigma}{\mathrm{d} d}=\left(\frac{1}{A}+\sum_{k=1}^{N} \frac{1}{B_{k}}\right)^{-1}
$$

Here,

$$
\sigma=\Theta^{\prime}(q)=\theta^{\prime}\left(q_{1}\right)=\cdots=\theta^{\prime}\left(q_{N}\right)
$$

is the overall equilibrium stress in the chain. Comparing Eq. (3.15) with Eq. (3.14) we obtain the following the stability criterion for a configuration with a single "concave" crack

$$
\frac{\mathrm{d} \sigma}{\mathrm{d} d} \leqslant 0 .
$$


This inequality means that for such a configuration to be metastable, the overall stress strain curve must have a negative slope. This result can be illustrated by the following example.

Consider a case when the bulk energy is quadratic,

$$
\Theta(q)=\frac{C}{2 l}(q-l)^{2}
$$

and the function $\theta$ has the bi-modal (concave-convex-concave) form shown in Fig. 1. It is convenient to introduce the average strain $\Delta=(d-l) / l$. Suppose first that $N=0$, so that $q_{k}=0$, and (from the constraint (3.7)) $q=d$. Then,

$$
E_{0}(\Delta)=\frac{1}{2} C l \Delta^{2}, \quad \sigma_{0}(\Delta)=C \Delta .
$$

Now, consider the case $N=1$. The equilibrium condition (3.8) reads

$$
\frac{C}{l}(q-l)=\theta^{\prime}\left(q_{1}\right)
$$

the constraint takes the form $q+q_{1}=d$, and we obtain a single non-linear equation for $q_{1}$

$$
C\left(\Delta-\frac{q_{1}}{l}\right)=\theta^{\prime}\left(q_{1}\right) \text {. }
$$

A solution of this equation can be obtained geometrically as an intersection of the family of parallel straight lines $z=C\left(\Delta-q_{1} / l\right)$, parameterized with $\Delta$ and $l$, with the graph of the function $z=\theta^{\prime}\left(q_{1}\right)$. The solution is in general a multi-valued function. After this function is found, the overall energy-strain and stress-strain relations can be obtained from the formulas

$$
E_{1}(\Delta)=\frac{C l}{2}\left(\Delta-\frac{q_{1}(\Delta)}{l}\right)^{2}+\theta\left(q_{1}(\Delta)\right), \quad \sigma_{1}(\Delta)=C\left(\Delta-\frac{q_{1}(\Delta)}{l}\right) .
$$

The functions $E_{0}, E_{1}, \sigma_{0}, \sigma_{1}$ are sketched in Fig. 2 for the case of sufficiently large $l$ (for the role of parameter $l$ see the discussion in Del Piero and Truskinovsky (1998)). The local minimizers are shown with bold lines. One can see that between points B and C, D and E, and F and G, the slope of the overall stressstrain curve is negative and the metastable equilibrium configuration contains a single crack from one of the "concavity" domains. On the other hand, between points C and D the equilibrium crack opening corresponds to the convex branch of the surface energy and the effective elastic modulus is positive.
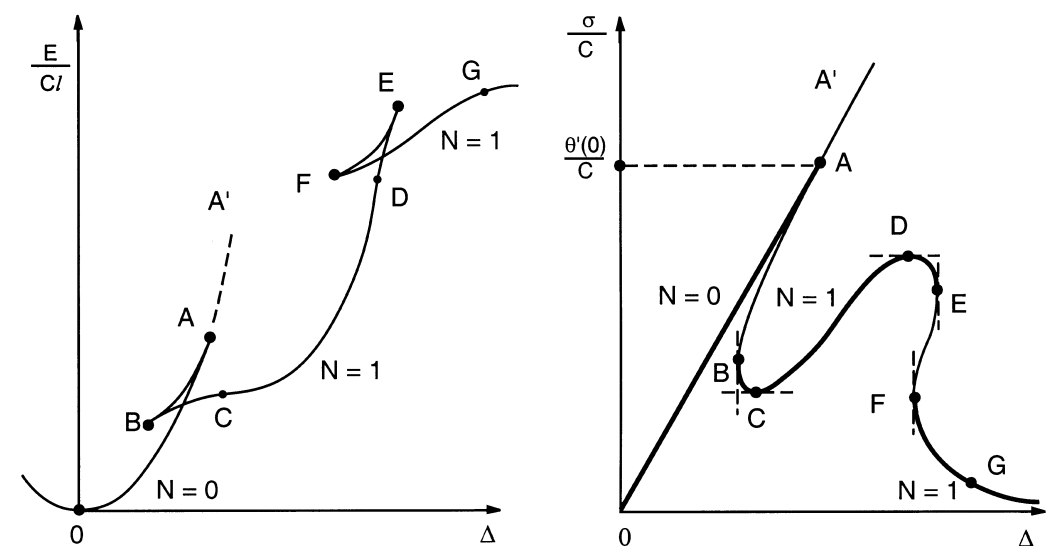

Fig. 2. Sketch of the metastable and unstable branches $N=0$ and $N=1$ for the case of quadratic bulk and bi-modal surface energies. 
Now let us return to the general problem of minimizing the energy with respect to $N$. We begin with the study of the bifurcations leading to the change of the number of jumps: in terms of the minimization problem at fixed $N$ the corresponding branching would mean that the minimum is achieved at the boundary of the domain of admissible $q_{k}$ (recall that $q_{k}>0$ ). It is clear that in this case the vanishing of the first variation is no longer necessary for equilibrium.

Suppose that a new infinitesimal crack appears in the bar without affecting the pre-existing cracks, so that the constraint (3.7) is accommodated by the bulk strain $q$. Then the variation of the energy (3.6) is given by

$$
\hat{E}\left(q-h, q_{k}, h\right)-\hat{E}\left(q, q_{k}\right)=\Theta(q-h)+\theta(h)-\Theta(q)=\left(-\Theta^{\prime}(q)+\theta^{\prime}(0)\right) h+o(h) .
$$

Since $h>0$ by Eq. (2.2), we conclude that for a configuration with a given number of cracks to be a local minimum the following inequality must hold

$$
\theta^{\prime}(0) \geqslant \Theta^{\prime}(q) \text {. }
$$

Together with the equilibrium conditions (3.8) this implies

$$
\theta^{\prime}(0) \geqslant \theta^{\prime}\left(q_{k}\right), \quad k=1, \ldots, N .
$$

In the following we restrict our attention to surface energies such that $\theta^{\prime}(0)>\theta^{\prime}([y])$ for all $[y]>0$ (see Fig. 1), so that the inequality (3.20) is always satisfied.

Condition (3.19) is necessary for an equilibrium configuration to be metastable. One can show that this condition (with strict inequality) together with equilibrium conditions (3.8) and stability conditions (3.10) (with strict inequality) is also sufficient for the metastability in the sense of the sup-norm: since the problem is essentially finite-dimensional, the formal proof is straightforward. We remark that if, instead, an integral norm is considered, the class of small perturbations is wider and sufficient conditions for metastability are expected to be stronger.

\section{Global minimization}

To find the global minimum one needs to consider finite perturbations and the local analysis is no longer sufficient. In particular, we need to compare the energies of the metastable branches at different $N$ 's, and for each value of the deformed length $d$ we have to determine the function $N^{*}(d)$ providing the number of cracks in the global minimizer. In this section, we show how the information on the global behavior of the function $\theta$ can be used to draw conclusions about the structure of the function $N^{*}$.

Certain issues have already been investigated in the literature. Thus, it is known that if $\theta$ is concave, one can have at most one crack: $N^{*}(d) \in\{0,1\}$ (see Truskinovsky (1996), Del Piero (1999) and Braides et al. (1999)). Also, if $\theta$ is convex or linear in the vicinity of the origin and if the bar is sufficiently short, $N^{*}(d)=\infty$ at least on a finite interval of $d$; in this case the cracks must obviously be infinitesimal and the minimum does not exist in the classical sense (see Braides and Coscia (1993) and Del Piero (1999)). We shall clarify these statements below; for now, our main goal is to find conditions on $\theta$ which guarantee that we can have discrete finite-scale cracking with $1<N^{*}(d)<\infty$.

We begin with the following definition (e.g. Braides and Coscia (1993)). We say that a function $\theta:[0, \infty) \rightarrow \mathbf{R}$ is subadditive if

$$
\theta(a) \leqslant \theta(\lambda a)+\theta((1-\lambda) a), \quad \forall \lambda \in(0,1) .
$$

This assumption means that it is never energetically favorable for a crack to split into smaller cracks with the same total opening. By letting $\lambda \rightarrow 0$, one can show that the condition of subadditivity (4.1) is stronger than the necessary condition of metastability (3.20) 


$$
\theta^{\prime}(0) \geqslant \theta^{\prime}(a), \quad a>0 .
$$

On the other hand, subadditivity is sufficient for the existence of a global minimizer. In fact, if $\theta$ is subadditive we obtain

$$
\tilde{E}\left(q_{k}\right)=\Theta\left(d-\sum_{k=1}^{N} q_{k}\right)+\sum_{k=1}^{N} \theta\left(q_{k}\right) \geqslant \Theta\left(d-\sum_{k=1}^{N} q_{k}\right)+\theta\left(\sum_{k=1}^{N} q_{k}\right) .
$$

This inequality guarantees that the absolute minimum is achieved at a configuration with

$$
N^{*}(d) \leqslant 1 \text {. }
$$

Accordingly, by introducing a new variable $Q=\sum_{k=1}^{N} q_{k}$ we may reduce Eqs. (3.6) and (3.7) to the onedimensional problem

$$
\bar{E}(Q)=\Theta(d-Q)+\theta(Q) \rightarrow \min , \quad 0 \leqslant Q \leqslant d .
$$

The existence of the minimum is now ensured by the continuity of $\bar{E}$ and by some obvious assumptions of growth for the bulk energy $w$.

The inequality (4.3) generalizes the result mentioned in the Introduction, that if the surface energy is concave, the number of cracks is either zero or one. This is not surprising, since concavity implies subadditivity: to check it, rewrite the inequality (4.1) as (recall $\theta(0)=0$ )

$$
\int_{a}^{a+b} \theta^{\prime}(q) \mathrm{d} q \leqslant \int_{0}^{b} \theta^{\prime}(q) \mathrm{d} q, \quad a, b \geqslant 0,
$$

and notice that for $\theta(a) \geqslant 0, \theta(0)=0$, concavity implies $\theta^{\prime}(b) \geqslant \theta^{\prime}(a)$ for $b \leqslant a$. On the contrary, a subadditive function is not necessarily concave on the whole domain, even if the necessary condition (4.2) requires concavity in the neighborhood of zero.

We notice that subadditivity in a neighborhood $[0, a]$ of the origin is also sufficient for the existence of a minimizer. In this case, all crack openings $q_{k}$ in the energy minimizer, except possibly one, satisfy $q_{k} \geqslant a / 2$. Consequently, instead of Eq. (4.3) we obtain the weaker restriction

$$
N^{*}(d) \leqslant 1+\frac{2 d}{a} .
$$

Since we are interested in modeling multiple micro-cracking $\left(N^{*}(d)>1\right)$, the surface energy cannot be subadditive on the whole interval, although it must have subadditive branches. Indeed, all cracks in a relative minimum must be located inside the domain of subadditivity of the function $\theta$, defined by

$$
\operatorname{sub} \theta=\left\{a>0, \quad \theta(a) \leqslant \sum_{i=1}^{m} \theta\left(a_{i}\right), \quad a_{i}>0, \quad \sum_{i=1}^{m} a_{i}=a, \quad m \in \mathbf{N}\right\} .
$$

To prove this fact, consider a finite perturbation changing the discontinuity set from $\left\{q_{1}, \ldots, q_{k}, \ldots, q_{N}\right\}$ into $\left\{q_{1}, \ldots, q_{k-1}, \eta_{1}, q_{k+1}, \ldots, q_{N}, \eta_{2}, \ldots, \eta_{m}\right\}$, with $\eta_{i}>0$ and $\sum_{i=1}^{m} \eta_{i}=q_{k}$. If the original configuration is a minimizer, we obtain the condition

$$
\tilde{E}\left(q_{l}, \eta_{j}\right)-\tilde{E}\left(q_{l}\right)=\theta\left(\eta_{1}\right)-\theta\left(q_{k}\right)+\sum_{i=2}^{m} \theta\left(\eta_{i}\right) \geqslant 0
$$

which can be rewritten as

$$
\theta\left(q_{k}\right) \leqslant \sum_{i=1}^{m} \theta\left(\eta_{i}\right)
$$


Hence $q_{k} \in \operatorname{sub} \theta$ due to the arbitrariness of $\eta_{i}$. It is important to realize that because the perturbations $\eta_{i}$ are not assumed to be small, the statement above does not apply to local minima in the sense of the sup-norm, which may well contain cracks outside the domain of subadditivity. On the other hand, if we consider local minimization in the sense of an integral norm, then even local minima must contain only "subadditive" cracks (see Del Piero and Truskinovsky (2000)).

To summarize, the domain of a non-subadditive surface energy must contain subadditive segments where the equilibrium discontinuities can be located. The situation here is somewhat similar to the case of one-dimensional theory of phase transitions, where the non-convexity of the bulk energy is necessary for the formation of phase mixtures, while the individual phases are always located on the convex branches of the energy function (see, for instance, Ericksen (1975)).

The "bi-modal" function $\theta$ shown in Fig. 1 is the simplest example of a surface energy for which multiple cracking is possible. The subadditivity of $\theta$ at the origin can not be sacrificed because this leads to the loss of lower semicontinuity of $E$, and therefore to diffuse damage $\left(N^{*}(d)=\infty\right)$. Suppose that $a^{*}$ is the infimum of $\operatorname{sub} \theta$. Then, any cracked configuration with $d<a^{*}$ is unstable. At the same time, the homogeneous uncracked configuration $q=d$ is stable only if $\Theta^{\prime}(d) \leqslant \theta^{\prime}(0)$, which, since $\Theta^{\prime}$ is monotonically increasing, means $d \leqslant a_{*}:=\Theta^{\prime-1}\left(\theta^{\prime}(0)\right)$. Now, if $a_{*}<a^{*}$ the minimum of $E$ does not exist for all $d$ in $\left(a_{*}, a^{*}\right)$. As shown in Del Piero (1999), the infimum of the energy can be associated with a "generalized" equilibrium configuration containing an infinite number of infinitesimal cracks. This configuration has a finite energy because for $q_{k}=d / N$

$$
\sum_{k=1}^{N} \theta\left(q_{k}\right)=N \theta\left(\frac{d}{N}\right)=\theta^{\prime}(0) d+o\left(N^{-1}\right) .
$$

The fact that the limiting energy depends linearly on the overall strain $d$ justifies the term "quasi-plasticity".

A systematic construction of the subadditivity domains for generic "bi-modal" surface energies and the evaluation of the corresponding bounds on the number of cracks will be given elsewhere (Del Piero and Truskinovsky (2000)). Here, we limit our consideration to an explicit example of a non-subadditive surface energy exhibiting a finite number $N^{*}(d)$ of equilibrium cracks.

\section{An example}

To illustrate the possibility of having a finite number of cracks, we consider here a very simple example. Assume again that the bulk energy is quadratic:

$$
w(p)=\frac{C}{2}(p-1)^{2}, \quad \Theta(q)=\frac{C}{2 l}(q-l)^{2} .
$$

For the "bi-modal" surface energy, we suppose that the first concave region near the origin is reduced to a point, while the second concave region at infinity is replaced by a constant; we also suppose that the intermediate convex region is represented by a quadratic function. Specifically, we take (Fig. 3)

$$
\theta(a)= \begin{cases}0, & a=0 \\ \gamma_{1}+A a+\frac{B^{2}-A^{2}}{4\left(\gamma_{2}-\gamma_{1}\right)} a^{2}, & 0<a<a_{*} \\ \gamma_{2}, & a \geqslant a_{*} .\end{cases}
$$

The parameters $\gamma_{1}, \gamma_{2}, A, B$ have a clear physical meaning. Indeed, $\gamma_{1}$ is the energy of the smallest opening (infinitesimal micro-crack) and $\gamma_{2}$ is the energy of a fully developed macro-crack. The stress barrier for the formation of the micro-crack is infinite, while the associated energy barrier for the creation of a macro- 

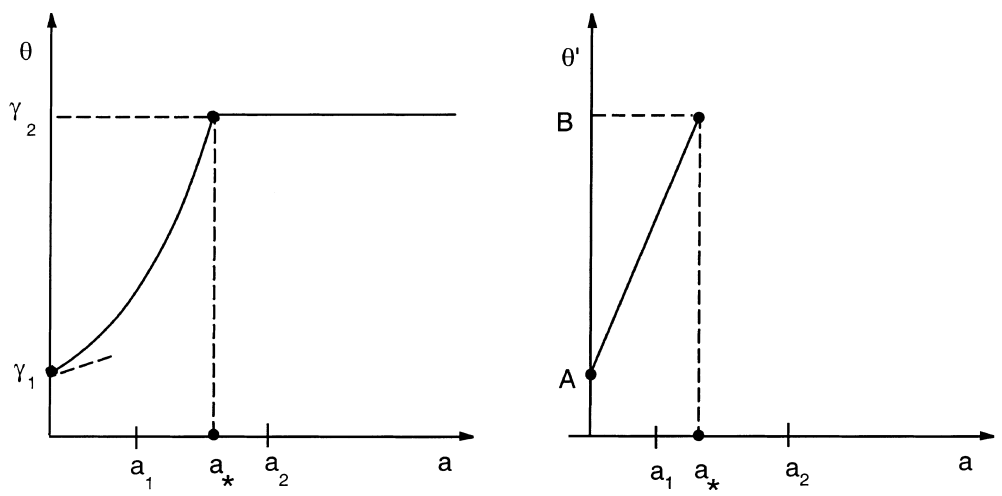

Fig. 3. Surface energy (5.2).

crack is finite; $A$ and $B$ measure the minimum and the maximum stress supported by a micro-crack before it develops into a macro-crack at

$$
a_{*}=\frac{2\left(\gamma_{2}-\gamma_{1}\right)}{A+B} .
$$

Since the concave regions in this model are degenerate, one expects an equilibrium configuration to contain either a finite number of micro-cracks located in the convex region or a single fully developed macro-crack with zero stress.

It is not hard to see that the function (5.2) is subadditive if the energy rate $\left(\gamma_{2}-\gamma_{1}\right) / \gamma_{1}$ is sufficiently small, more specifically, if

$$
\frac{\gamma_{2}-\gamma_{1}}{\gamma_{1}}<2 \frac{B+A}{B-A}
$$

If this condition is not satisfied, the domain of subadditivity can be shown to consist of two segments $0 \leqslant a \leqslant a_{1}$ and $a_{2} \leqslant a<\infty$, where

$$
\begin{aligned}
& a_{1}=2\left(\frac{2\left(\gamma_{2}-\gamma_{1}\right) \gamma_{1}}{B^{2}-A^{2}}\right)^{\frac{1}{2}}, \\
& a_{2}=2(N+1) \frac{\gamma_{2}-\gamma_{1}}{B^{2}-A^{2}}\left[-A+\left(A^{2}+\left(\frac{\gamma_{2}}{N+1}-\gamma_{1}\right) \frac{B^{2}-A^{2}}{\gamma_{2}-\gamma_{1}}\right)^{\frac{1}{2}}\right],
\end{aligned}
$$

with $N$ equal to the integer part of

$$
\frac{1}{2} \frac{B^{2}\left(\gamma_{2}-\gamma_{1}\right)}{B^{2} \gamma_{1}-A^{2} \gamma_{2}}\left[1-\left(1-\frac{\left(B^{2}-A^{2}\right)\left(B^{2} \gamma_{1}-A^{2} \gamma_{2}\right)}{B^{4} \gamma_{1}}\right)^{\frac{1}{2}}\right] .
$$

The non-subadditivity of the surface energy suggests that the minimizers may have more than one microcrack and the analytical simplicity of the model allows one to determine the multi-crack configurations explicitly.

We first notice that there are three types of metastable configurations. First, a homogeneous, undamaged configuration with all $q_{k}=0$. The overall energy and stress in this configuration are given by 


$$
E_{0}(d)=\frac{C}{2 l}(d-l)^{2}, \quad \sigma_{0}(d)=\frac{C}{l}(d-l)^{2} .
$$

Due to our specific choice of the function $\theta$, condition (3.19) is trivially satisfied and this configuration is metastable for all $d \geqslant l$.

The second special case refers to an unstressed configuration with a fully developed macro-crack. In this situation the overall energy and stress are given by

$$
E_{1}(d)=\gamma_{2}, \quad \sigma_{1}(d)=0 .
$$

Finally, there are configurations with $N>1$ micro-cracks. From the equilibrium condition (3.8) one can see that the corresponding openings must be all the same: $q_{k}=\bar{q}, k=1, \ldots, N$. Then the elastic energy can be written as

$$
\tilde{E}_{N}(q, \bar{q})=\Theta(q)+N \theta(\bar{q})
$$

where the variables $q$ and $\bar{q}$ must satisfy the constraint

$$
q+N \bar{q}=d .
$$

Since the functions $\theta$ and $\Theta$ are known, the equilibrium condition (3.8) can be written in the explicit form

$$
q=l+\frac{A l}{C}+\frac{l\left(B^{2}-A^{2}\right)}{2 C\left(\gamma_{2}-\gamma_{1}\right)} \bar{q}
$$

Application of the hard device (5.6) gives

$$
\bar{q}=\frac{d-l-\frac{A l}{C}}{N+\frac{l\left(B^{2}-A^{2}\right)}{2 C\left(\gamma_{2}-\gamma_{1}\right)}}, \quad q=l+\frac{A l}{C}+\frac{d-l-\frac{A l}{C}}{1+N \frac{2 C\left(\gamma_{2}-\gamma_{1}\right)}{l\left(B^{2}-A^{2}\right)}} .
$$

Now it is easy to calculate the overall stress. Recall that $\sigma=\Theta^{\prime}(a)=C(q-l) / l$ by Eq. (5.1). Then

$$
\tilde{\sigma}_{N}(d)=A+\frac{d-l-\frac{A l}{C}}{\frac{l}{C}+N \frac{2\left(\gamma_{2}-\gamma_{1}\right)}{\left(B^{2}-A^{2}\right)}} .
$$

To obtain an explicit expression for the total energy, we start with the implicit relation

$$
\tilde{E}_{N}(d)=\frac{C}{l}(q-l)^{2}+N\left(\gamma_{1}+A \bar{q}+\frac{\left(B^{2}-A^{2}\right)}{4\left(\gamma_{2}-\gamma_{1}\right)} \bar{q}^{2}\right),
$$

which, after introducing the non-dimensional parameters

$$
P=\frac{A}{C}, \quad R=\frac{l\left(B^{2}-A^{2}\right)}{2 C\left(\gamma_{2}-\gamma_{1}\right)}, \quad \Gamma_{1}=\frac{\gamma_{1}}{C l}, \quad \Gamma_{2}=\frac{\gamma_{2}}{C l},
$$

we rewrite in the form

$$
\frac{\tilde{E}_{N}(\Delta)}{C l}=\frac{1}{2} P^{2}+N \Gamma_{1}+P(\Delta-P)+\frac{1}{2} \frac{R}{R+N}(\Delta-P)^{2} .
$$

Here, $\Delta=(d-l) / l$ is again the total strain. From the expression (5.12) one can see that the equilibrium branches corresponding to different $N$ are represented on the $E-\Delta$ plane by intersecting parabolas. The overall stress-strain relation for these metastable configurations can now be represented on the $\sigma-\Delta$ plane by a set of straight lines

$$
\frac{\tilde{\sigma}_{N}(\Delta)}{C}=P+\frac{R}{R+N}(\Delta-P) .
$$



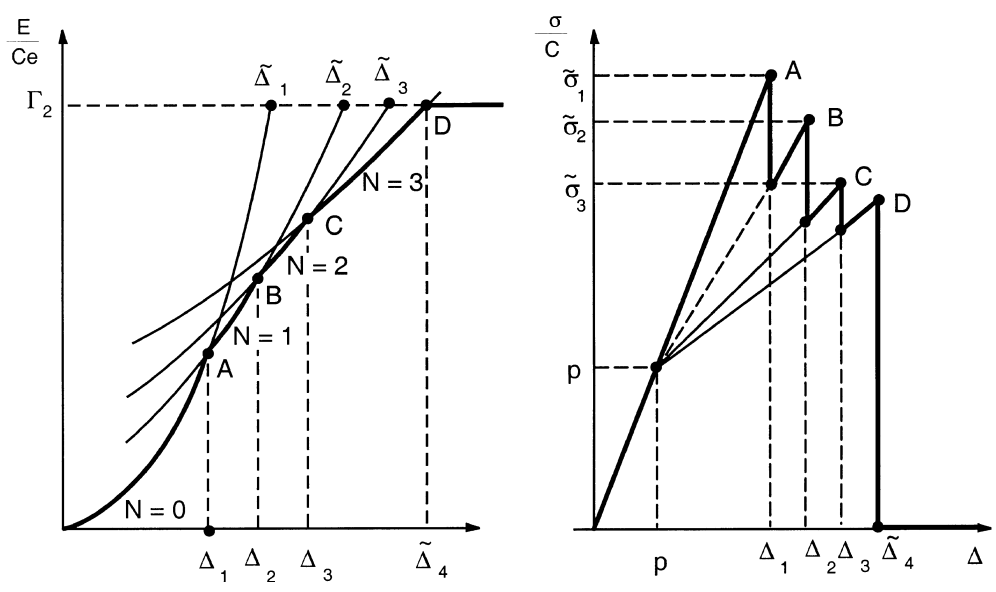

Fig. 4. Total energy and overall stress-strain relations for the stable (global minimum) and metastable (local minimum) configurations in the model with surface energy (5.2).

To find the global minimum of the energy, we need to specify points where the energy branches corresponding to different number of cracks intersect. By using Eq. (5.12), we obtain that the branch with $N$ micro-cracks intersects the branch with $N+1$ micro-cracks at

$$
\Delta_{N}=P+\left[\frac{2 \Gamma_{1}}{R}(N+R)(N+R+1)\right]^{\frac{1}{2}} .
$$

The dependence of energy and stress upon the total elongation is shown qualitatively in Fig. 4, where only the metastable branches are drawn.

From Fig. 4 we see that as the average strain increases, the energy minimizing configurations have increasingly large number of cracks, starting from $N=1$ and with more cracks forming until the mode $N=1$ prevails once again. The stress drops with the formation of every new crack. As we show below, the total number of the micro-cracks formed before the occurrence of the final failure depends on the length of the bar.

Notice that at the "Maxwell" stresses

$$
\frac{\tilde{\sigma}_{N}}{C}=P+\left[2 \Gamma_{1} R \frac{N+R+1}{N+R}\right]^{\frac{1}{2}}
$$

where the structure of the global minimizer changes, a new micro-crack forms and the stress drops in the amount

$$
[\tilde{\sigma}]_{N}=\left[\frac{2 \Gamma_{1} R}{(N+R+1)(N+R)}\right]^{\frac{1}{2}}
$$

The magnitude of stress drop decreases with $N$. To determine the value of $d$ at which the ultimate macrocrack forms, we need to compare the energies of the above metastable configurations with the energy of the "macro-cracked" solution (5.4). The branch with $N$ micro-cracks intersects the branch (5.4) at

$$
\tilde{\Delta}_{N}=\frac{R+N}{R}\left[-\frac{P N}{R+N}+\left(\frac{P^{2} N}{R+N}+\frac{2\left(\Gamma_{2}-N \Gamma_{1}\right) R}{R+N}\right)^{\frac{1}{2}}\right] .
$$


It is now clear from Fig. 4 that the condition $\Delta_{N} \leqslant \tilde{\Delta}_{N}$ defines the maximum number of micro-cracks in the global minimizer. Written explicitly, this condition reads

$$
\left[P^{2}(N+R)\right]^{\frac{1}{2}}+\left[2 \Gamma_{1} R(N+R+1)\right]^{\frac{1}{2}} \leqslant\left[P^{2} N+2\left(\Gamma_{2}-N \Gamma_{1}\right) R\right]^{\frac{1}{2}}
$$

If $N$ is the largest integer satisfying this inequality, the maximum number of micro-cracks is equal to $N+1$.

The analysis of the above solution suggests the following scenario for the propagation of fracture. The undamaged state of the bar is stable until a certain critical elongation is achieved. Then micro-defects start to form, one after another. For a given $\Delta$, there may be several metastable configurations with a different number of micro-cracks, although the global minimum includes a specific number of "defects". At sufficiently large elongations, the global minimum is always achieved at a configuration with a single macrocrack.

Finally, we notice that in our example failure propagates in a discrete manner, despite the fact that the original problem contains no elements of discreteness. A similar "quantization" was observed in Truskinovsky and Zanzotto (1996) for shape memory bars on elastic foundation, where the role of defects was played by phase boundaries.

\section{Acknowledgements}

This research has been supported by the Programma Nazionale Cofinanziato 1998, "Modelli Matematici per la Scienza dei Materiali" and by the NSF grants DMS 9501433 and 9803572.

\section{References}

Baljon, A.R.C., Robbins, M.O., 1996. Energy dissipation during rupture of adhesive bonds. Science 271, $482-484$.

Barenblatt, G.I., 1962. The mathematical theory of equilibrium cracks in brittle fracture. Adv. Appl. Mech. 7, 55-129.

Braides, A., Coscia, A., 1993. A singular perturbation approach to variational problems in fracture mechanics. Math. Mod. Meth. Appl. Sci. 3, 303-340.

Braides, A., Dal Maso, G., Garroni, A., 1999. Variational formulation of softening phenomena in fracture mechanics: the onedimensional case. Arch. Rat. Mech. Anal. 146, 23-58.

Del Piero, G., 1999. One-dimensional ductile-brittle transition, yielding, and structured deformations. In: Argoul, P., Fre'mond, M., Nguyen, Q.S., (Eds.).Variations de domaines et frontières libres en mécanique des solides. Kluwer, Dordrecht.

Del Piero, G., Owen, D.R., 1993. Structured deformations of continua. Arch. Rat. Mech. Anal. 124, 99-155.

Del Piero, G., Truskinovsky, L., 1998. A one-dimensional model for localized and distributed fracture. J. de Physique IV 8, 95-102.

Del Piero, G., Truskinovsky, L., 2000. Variational analysis of multiple cracking, to be submitted.

Ericksen, J.L., 1975. Equilibrium of bars. J. Elast. 5, 191-201.

Griffith, A.A., 1920. The phenomenon of rupture and flow in solids. Phil. Trans. Roy. Soc. London A 221, $163-198$.

Kachanov, M., 1993. Elastic solids with many cracks and related problems. Adv. Appl. Mech. 30, $59-445$.

Puglisi, G., Truskinovsky, L., 2000. Mechanics of a discrete chain with bi-stable elements. J. Mech. Phys. Solids. 48, 1-27.

Truskinovsky, L., 1996. Fracture as a phase transition. In: Batra, R.C., Beatty, M.F. (Eds.). Contemporary research in the mechanics and mathematics of materials. CIMNE, Barcelona, pp. 322-332.

Truskinovsky, L., Zanzotto, G., 1996. Ericksen's bar revisited: energy wiggles. J. Mech. Phys. Solids 44, 1371-1408. 